\title{
Ensayo
}

Pensar en Movimiento:

Revista de Ciencias del Ejercicio y la Salud ISSN 1659-4436

Vol. 15, No.2, pp. 1- 12

Abre el $1^{\circ}$ de julio, cierre al 31 de diciembre, 2017

\section{PENSAMIENTO DECIMONÓNICO CUBANO: CONTRIBUCIÓN A LA EDUCACIÓN FÍSICA Y EL DEPORTE}

\author{
CUBAN NINETEENTH-CENTURY THOUGHT: CONTRIBUTION TO PHYSICAL \\ EDUCATION AND SPORTS
}

\section{PENSAMENTO DECIMONÔNICO CUBANO: CONTRIBUIÇÃO PARA A EDUCAÇÃO FÍSICA E O ESPORTE}

\author{
Reynier Rodríguez-González, Ph.D. \\ rrodriqueza@ups.edu.ec \\ Universidad Politécnica Salesiana, Guayaquil, Ecuador \\ Envío original: 24/06/2017; reenviado: 15/09/2017; \\ aceptado: 14/11/2017; publicado: 13/12/2017.
}

Doi: 10.15517/pensarmov.v15i2.29590

\section{RESUMEN}

Rodríguez-González, R. (2017). Pensamiento decimonónico cubano: contribución a la educación física y el deporte. Pensar en Movimiento: Revista de Ciencias del Ejercicio y la Salud, 15(2), 1-12. El objetivo de este artículo es profundizar en las relaciones existentes entre la formación humanista y la Cultura Física en el pensamiento pedagógico cubano del siglo XIX. Con este fin, se realizó una revisión bibliográfica de las publicaciones de autores relevantes que han abordado el tema y de aquellas que se refieren a las fuentes de pensamiento, para una mejor interpretación y valoración del problema. Se constató el sentido integrador, selectivo y crítico del pensamiento pedagógico cubano de esa época en su carácter sociocultural universal y autóctono, manifestado en acciones diversas para introducir la formación humanista y la Cultura Física en el contexto formativo. Los resultados muestran las insuficiencias en el tratamiento actual de los aspectos relacionados con la Cultura Física 
en la historiografía cubana y la escasa profundización en los aportes de los pedagogos cubanos del siglo XIX a la formación humanista y su estrecha relación con la Cultura Física. Se proponen futuras líneas de investigación como posible contribución a la formación de licenciados en dicha área.

Palabras claves: pensamiento pedagógico; historia de Cuba; historia de la Cultura Física; humanismo; formación humanista; Cultura Física.

\begin{abstract}
Rodríguez-González, R. (2017). Cuban Nineteenth-Century Thought: Contribution to Physical Education and Sports. Pensar en Movimiento: Revista de Ciencias del Ejercicio y la Salud, 15(2), 1-12. The objective of this article is to explore the existing relationships between the humanistic formation and physical culture in the Cuban pedagogical thinking during the nineteenth century. With this goal in mind, a bibliographical search was performed including the most relevant authors who had addressed the topic and including some sources of their thinking, in order to better interpret and assess this topic. The integrative, selective and critical approach of Cuban pedagogical thinking from that era was verified in its sociocultural, universal, and autochthonous character, patent in different actions meant to introduce the humanistic model and physical culture into the formative process. We show some shortcomings in the present approach to those elements associated with physical culture in Cuban history, and the lack of solid discussion about $19^{\text {th }}$ century Cuban teachers' contributions to the humanistic model and its close relation to physical culture. Future research topics are proposed as a possible contribution to training professionals in this field.
\end{abstract}

Key Words: pedagogical thinking; Cuban history; physical culture history; humanism; humanistic model; physical culture

\title{
RESUMO
}

Rodríguez-González, R. (2017). Pensamento decimonônico cubano: contribuição para a educação física e o esporte. Pensar en Movimiento: Revista de Ciencias del Ejercicio y la Salud, 15(2), 1-12. O objetivo deste artigo é aprofundar nas relações existentes entre a formação humanista e a Cultura Física no pensamento pedagógico cubano do século XIX. Com esse fim, realizou-se uma revisão bibliográfica das publicações de autores relevantes que abordaram o tema e daquelas que se referem às fontes de pensamento, para uma melhor interpretação e avaliação do problema. Constatou-se o sentido integrador, seletivo e crítico do pensamento pedagógico cubano dessa época em seu carácter sociocultural universal e autóctone, manifestado em diversas ações para introduzir a formação humanista e a Cultura Física no contexto formativo. Os resultados mostram as insuficiências no tratamento atual dos aspectos relacionados com a Cultura Física na historiografia cubana e o escasso aprofundamento nas contribuições dos pedagogos cubanos do século XIX à formação humanista e sua estreita relação com a Cultura Física. Propõem-se futuras linhas de investigação como possível contribuição para a formação de licenciados em tal área. 
Palavras-chaves: pensamento pedagógico; história de Cuba; história da Cultura Física; humanismo; formação humanista; Cultura Física

En el siglo XIX, los pensadores latinoamericanos propiciaron un cambio en el pensamiento filosófico y científico, con su constante contribución al desarrollo científicotécnico, el progreso de los métodos del conocimiento y la oposición a la escolástica. Además, propusieron ideas orientadas a la enseñanza del pensar y el sentir, sobre la base de una ética comprometida con la naciente conciencia americana (Guadarrama, 2001).

En las obras de diferentes pensadores cubanos en la época de la colonia se constata la preocupación por el desarrollo de una personalidad armónica. La necesidad de educar, a partir del desarrollo de todas las potencialidades, hizo que se considerara en diferentes ámbitos de la vida colonial la inclusión de la educación física y los deportes, por la naturaleza educativa de ambas prácticas (Rodríguez-González, 2010, 2016).

En el período colonial, concretamente en el siglo XIX, hay antecedentes sobre el origen y el desarrollo de la Cultura Física. Sin embargo, en la historiografía educativa cubana este tema aún no se ha tratado teórica y metodológicamente con la intensidad y la profundidad que las ciencias sociales contemporáneas requieren, lo cual se comprueba en algunos textos de carácter histórico. En otros, como la colección "Historia de la educación en Cuba" (Sosa \& Penabad, 2005a, 2005b), el tratamiento es muy exiguo, lo que confirma, de forma general, el poco interés por esta temática en lo académico y lo científico en las investigaciones sobre la Cultura Física, salvo las contribuciones de Sánchez (2004) y Salas (2009).

No obstante, en la obra de los pensadores del siglo XIX y en una serie de hechos históricos que contribuyeron en este sentido, existen referencias sobre el tema. Es importante señalarlo por la tendencia eurocéntrica a desestimar los valores del pensamiento filosófico latinoamericano, enmarcándolo en lo academicista y desvinculándolo de cualquier interés por los problemas sociales. Por tanto, es significativo descubrir el pensamiento pedagógico de este período como una forma de expresión de la singularidad en la universalidad. En esta época se observa una clara influencia de diferentes pensadores europeos cuyas enseñanzas propagaron vertiginosamente las ideas sobre el ejercicio físico, en primer lugar, y el deporte, posteriormente (Rodríguez-González, 2011a, 2011b). También se desarrolló un gran interés pedagógico por la educación física como medio para la formación humanista. Aún así, las investigaciones sobre los estudios de pensamiento son insuficientes en relación con las influencias de estos pensadores en el origen y el desarrollo de la Cultura Física en Cuba. En particular las relacionadas con Vives $(\underline{1988}, \underline{1994}, \underline{1997})$, ignorado en algunos textos de Historia de la Cultura Física, aun cuando en su obra se constatan claras alusiones a temas de la cultura física.

\section{ANTECEDENTES}

En el siglo XIX aparecen análisis intelectuales sobre temas relacionados con la Cultura Física; sin embargo, no son referidos o analizados en textos relacionados con la historia de la Cultura Física, como los de Masjuan (1984), Salas (2009), Mariño (2011) y Martínez (2006). Por solo nombrar dos ejemplos: el interés por el Olimpismo se reflejó en el Papel Periódico de La Habana, con el artículo del periódico Mercurio Peruano, titulado "Examen Histórico de las Naciones", tomado del periódico Mercurio Peruano, el cual ubica la aparición del teatro griego primero que los Juegos Olímpicos. El pensador cubano Romay refutó estos criterios en otro 
artículo, al plantear que el proceso en Grecia fue a la inversa: hizo referencia a Ifitos, Coiracebus y Heracles y a leyendas sobre el inicio de los Juegos Olímpicos y expresó, además, sus conocimientos sobre las bondades de la actividad física al abordar la importancia de los ejercicios. Por otra parte, en su texto Elogio al Excelentísimo señor Luis de las Casas y Aragón, leído a los miembros de la Sociedad Económica Amigos del País el 19 de mayo de 1801, criticó el desprecio hacia los ejercicios atléticos y habló sobre la incorporación de aspectos relacionados con la educación física y moral para la formación (Mariño, 2011).

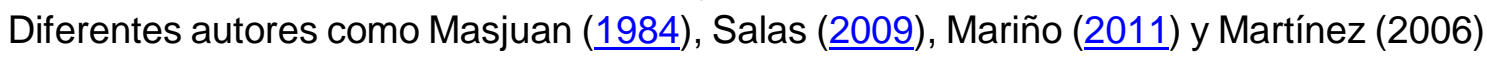
no consideran en sus textos los comentarios positivos de Romay sobre el sistema educativo de Pestalozzi. Aun cuando este autor, sin referirse a la educación física explícitamente, indica su inclinación por los nuevos avances pedagógicos; tan es así que nombra al pedagogo español Amorós entre las personalidades que estuvieron en la inauguración de la Real Escuela de Pruebas de Madrid.

Otro hecho poco recogido en textos históricos referidos a la Cultura Física es que en el Regañón del 5 de enero de 1802, Romay también expresó la necesidad de la formación física para las mujeres y lo ejemplificó con la mujer espartana, por su patriotismo y robustez. Posteriormente, reiteró la importancia de los ejercicios físicos para la salud y la longevidad. Argumentó que la pereza no contribuía a llegar sano a la vejez, mientras los ejercicios alargaban la vida con el aumento de los espíritus vitales y retardaban el proceso de envejecimiento con la restauración y endurecimiento de los órganos (Mariño, 2011).

Un hecho poco documentado es el que se vincula con Céspedes, gran apasionado por el juego ciencia, quien tradujo del francés al español el libro Las leyes del Juego de Ajedrez, del célebre maestro Charles de Labourdonnais, publicado el 4 de octubre de 1855 en el periódico El Redactor, de Santiago de Cuba.

También son insuficientes los análisis de cómo las diferentes tendencias de pensamientos que se fueron desarrollando en Cuba influyeron en la inclusión de la educación física y el deporte en la isla (Sánchez, 2004). Entre las corrientes de pensamiento del siglo XIX se destacó la llustración Reformista Cubana, surgida hacia 1802, con un campo de acción mayor que el movimiento de la Generación del 92. Fue una tendencia política y sociocultural, encabezada por el obispo Díaz de Espada, considerada por ciertos sector sociales como equívoca, a pesar de ser la que más incidió en la lucha por la independencia nacional antes de 1868.

Este movimiento tuvo dos centros irradiadores de la cultura y el pensamiento cubanos, el Real y Conciliar Seminario de San Carlos y San Ambrosio y la Real Sociedad Económica Amigos del País, ambos bajo la dirección intelectual y espiritual del obispo Díaz de Espada. Sobresalen el proyecto ilustrado y las acciones sociales del prelado que, a partir de su cargo como director, promovió en 1793 a intelectuales de la Real Sociedad Económica Amigos del País. Su obra tiene una significación considerable para la formación cultural y la identidad nacional. También es fundacional y revolucionaria porque impulsó la creación de un seminario en La Habana, el cual contribuyó a la formación del pensamiento iluminista en Cuba. Esta iniciativa recibió la influencia de pensadores como fray Feijoó, quien estremeció todo el basamento del quietismo español de la Edad Media con su exhortación teológica a conjugar las ciencias modernas con la religión. Si se ignoran estos antecedentes, sería imposible comprender la asunción de un sistema moderno de enseñanza acorde con lo propuesto por Pestalozzi. 


\section{El pensamiento pedagógico, sustento de la Educación Física y el deporte}

También hicieron valiosos aportes a la pedagogía José Agustín Caballero, Félix Varela y José de la Luz y Caballero, quienes no solo fueron los mayores exponentes del humanismo cristiano en el siglo XIX, al otorgarle singularidades propias como la estrecha relación entre fe y el amor a la patria, sino que también hicieron valiosos aportes a la pedagogía, al renovarla sustancialmente en su desarrollo, paralelo al proyecto educativo de la baja escolásticahispana. En este sentido, promovieron la incorporación de novedosos criterios en el campo de esta ciencia, aparejados a nuevas ideas sobre la física experimental y un nuevo deleite por lo neoclásico, sobre todo en el cambio de las concepciones teóricas.

Caballero desempeñó un papel importante como promotor de cambios culturales al establecer una distinción significativa con la etapa anterior en la enseñanza de la filosofía, distinción considerada como la búsqueda de un itinerario propio de la misma en Cuba. Su obra Philosophia electiva intentó sondear la postura ante el conocimiento como consecuencia del cambio de la forma de pensar y la autovaloración en relación con las competencias del criollo. Como pedagogo, abogó por una reforma de la enseñanza, porque era retrógrada y no favorecía el establecimiento de bases científicas acordes con los avances de la época. El método electivo, su aporte esencial, constituye una manifestación singular para adquirir e interrelacionar eficazmente nuevos conocimientos, así como la propuesta de la experimentación como método para el conocimiento de la naturaleza.

Con Varela, el pensamiento filosófico adquirió un nuevo matiz. La lógica interna de su pensamiento filosófico se orientó hacia un objetivo fundamental: la formación de las nuevas generaciones a través del ejercicio de pensar por sí mismos, la virtud y la piedad. Por tanto, no se puede separar su pensamiento filosófico de su pensamiento pedagógico, y menos aún de su pensamiento teológico que atraviesa a ambos, aunque es el menos estudiado con sistematicidad actualmente. Su pensamiento fue uno de los más elaborados en correspondencia con el humanismo pedagógico latinoamericano de la época y enriqueció la Pedagogía con la introducción de la enseñanza de las ciencias y el método experimental, el fomento de la racionalidad, el establecimiento de criterios sobre la educación infantil, la defensa del uso de la lengua materna y la promoción de la educación popular (Torres, 2006a). Propuso, además, la observación de la naturaleza y de los aciertos y desaciertos del hombre en la historia, para desarrollar el análisis como operación lógica del pensamiento; criticó los inconvenientes de la enseñanza memorística y la completa desvinculación de algunas materias con la práctica; sustituyó el latín por el castellano en las clases y escribió en su propio idioma varios textos filosóficos para la enseñanza (Varela y Morales, 2001a; 2001b; 2001c).

Su pensamiento está impregnado de sus ideas sobre el ser humano y la estabilidad social y de su inquietud por la plenitud de la persona en una sociedad nueva cada vez más justa y libre. El contenido ético del pensamiento vareliano tiene una significación extraordinaria, porque comprendió que no bastaba razonar bien, sino que también se debía despertar y desarrollar la conciencia moral desde los primeros años de vida.

Como filósofo, abogó por conjugar la teoría con la práctica en la enseñanza. Expuso la importancia del método teórico-práctico, en particular en la enseñanza del latín, y señaló lo aberrante de enseñarlo sin práctica, traducción ni explicación algunas. El modo de enseñar un idioma, tal como él lo concibió, es importante actualmente para la formación humanista desde lo didáctico, lo metodológico y lo científico; válido incluso para la enseñanza de la 
lengua materna y las extranjeras. Sus criterios sobre la enseñanza de las lenguas clásicas forma parte de la influencia recibida por corrientes del pensamiento europeo, en particular el neohumanismo, que supo adecuar al contexto formativo.

El método electivo, utilizado por Varela, es fundamental para la historia del pensamiento porque surgió ante la necesidad ineludible de cambiar el paradigma del conocimiento eurocéntrico y aunar la teoría y la historia del pensamiento universal, a partir de una actitud electiva, que contribuyera a la búsqueda de un camino propio para expresar la realidad insular, apartado de los esquemas inherentes al escolasticismo y su dependencia de las normas de Europa, a fin de promover el surgimiento, necesario, por otra parte, de una nueva forma de pensamiento que reuniera en sí los componentes físicos, culturales y éticos de la germinal y todavía no definida cubanidad (Torres, 2006b).

Varela propuso un método en el que la razón era indispensable para el razonamiento filosófico y la educación. Dirigió su atención a la teoría del conocimiento para analizar este proceso con la misma imparcialidad con que otro trata su propio objetivo de análisis; pensaba que el conocimiento era un proceso natural, por tanto, sus particularidades se podían aplicar de acuerdo con los métodos de las ciencias. Defendió la experimentación y el análisis, e implementó el método experimental en clase, lo cual fue uno de sus aportes a la pedagogía.

Cartas a Elpidio representa un total florecimiento de su pensamiento sociopolítico. En esta obra educativa no convencional se percibe un fuerte contenido axiológico y se plantea la subordinación del bien individual al bien común. Su pensamiento humanista también se destacó en la defensa de la abolición de la esclavitud, en el criterio de la verdad, al exponer que la persona no debe abandonar su camino hacia la justicia ni el atinado ímpetu que la dirige hacia la verdad; además, es notable su concepción sobre la perfección humana, al decir que el desarrollo del hombre se puede orientar cada vez más hacia la excelencia. Consideró la educación y la moralidad como medios para formar por la razón y el buen ejemplo una sociedad de hombres virtuosos.

En este período también se destacó por su enseñanza Luz y Caballero, quien recibió una gran influencia del humanismo cristiano de Varela. Su vasta obra en la primera mitad del siglo XIX puede verse, sin lugar a dudas, como una de las expresiones cimeras de la filosofía educativa, lo que lo convierte en un símbolo del magisterio cubano. Se caracterizó por utilizar la educación como una vía para la formación humanista; en particular, dirigió su obra a la formación de la conciencia ciudadana de las nuevas generaciones. Diseñó una estrategia en la que consideró la pedagogía como la ciencia de la enseñanza, convirtiendo la escuela en centro de formación de las nuevas generaciones; ajustó la enseñanza a las condiciones históricas concretas; tomó lo mejor del pensamiento pedagógico universal y le imprimió un sello propio; concibió la educación en un sentido integral y como responsabilidad de todos; y estimó en grado sumo la formación docente.

Asimismo, este autor introdujo el método explicativo para desarrollar la reflexión y criticó el método de memorización mecánica. Es fundamental señalar su énfasis en la unidad inseparable entre la instrucción y la educación; concebía la educación como la base única de las reformas sociales. Para él, educar no era solo trasmitir conocimientos sobre gramática, geografía y física, sino fortalecer el espíritu y hacer que se pusieran de relieve las mejores cualidades de la inteligencia y el carácter en el niño. Además, recomendó el método del premio y el del buen ejemplo para la formación humanista; el último es uno de los sugeridos para la formación humanista de los estudiantes. Consideró que el primer deber de los profesores era dar un buen ejemplo, porque era más efectivo que las normas mejor expresadas y las palabras 
más sugerentes; hay que enseñar buenos principios y hábitos, pues las palabras no son suficientes para educar, hay que unir la acción a la palabra. Igualmente, señaló cómo los estudiantes son muy observadores de la conducta de los profesores y expresó que no hay costumbre o actitud que no se pueda transformar cuando se muestra a los estudiantes el valor de las buenas acciones a través del ejemplo. También señaló la necesidad de que los estudiantes no solo escucharan hablar sobre la virtud, sino que también la observaran en la práctica en sus formadores (Luz y Caballero, 2001c).

En el período educativo comprendido entre 1800 y 1842 hubo un elevado interés por el aprendizaje, de forma paulatina, de la esgrima y la equitación en algunas escuelas y, posteriormente, en la mayoría de los grandes planteles de educación primaria y secundaria, a pesar de no practicarse regularmente como método de enseñanza. Lo anterior se comprueba en la obra de Luz y Caballero, quien formuló la necesidad de desarrollar la inteligencia a la par de la educación física; al respecto, se destacó como apologista de la misma y fundador del primer gimnasio cubano. Desde el primer momento se vinculó a esta institución y favoreció la práctica de ejercicios físicos en su colegio, "El Salvador". Además, expuso la importancia de la educación física en los primeros años de vida para la formación posterior de la juventud (Luz y Caballero, 2001b).

Clasificó las facultades del hombre en físicas, intelectuales y morales, y la educación en tres ramas: educación física, intelectual y moral. Abordó, el principio de la formación integral del ser humano al referirse a la necesidad de la formación de todas las facetas que conforman la personalidad, dedicándole su atención a cada una de ellas (Luz y Caballero, 2001a). Este principio sienta las bases de una correcta formación humanista, al considerar al hombre en su conjunto y en su singularidad y, por ende, presta atención al desarrollo equilibrado de sus capacidades físicas y espirituales, ya que, de no hacerlo, se afectaría su futuro al desatender una en beneficio de la otra y se negarían los propósitos más auténticos de la naturaleza y la sociedad. Como se puede apreciar, su obra está permeada por un alto grado de humanismo pedagógico, y es significativo su apego al magisterio como fuente de formación del hombre, en correspondencia con el desarrollo de cada una de las dimensiones que lo configuran como un todo indivisible.

Otro de los pensadores que valoró positivamente los beneficios del ejercicio físico fue Saco. En su autobiografía comentó que en las vacaciones iba con su padre al campo y allí practicaba actividades físicas tales como saltar, correr, nadar y montar a caballo, entre otras; las que al fortalecerlo físicamente le ayudaron a tener una vida prolongada. Para este pensador la actividad física mejora la calidad de vida de las personas. Fue un excelente científico social y censor de las lacras del colonialismo; criticó el juego aparejado al vicio, porque atenta contra el desarrollo pleno del ser humano y la sociedad. Su pensamiento influyó en el proceso de formación de la nación y la nacionalidad y realizó uno de los primeros análisis sociológicos detallados de las causas de la propagación y los efectos de los juegos (Saco, 2001).

Fue el iniciador de la crítica social en Cuba, arremetió a través de un profundo estudio sociológico contra los vicios y los juegos de azar en los cafés, justificados en ciertas festividades por la desidia del gobierno y las clases pudientes que aceptaban y hasta fomentaban esa situación, aunque en realidad degradaban al pueblo. Fue uno de los pensadores cubanos que enriqueció extraordinariamente la sociológica y política de los simpatizantes del pensamiento ilustrado (Saco, 2001). 


\section{Fundación del primer gimnasio cubano}

También fueron relevantes otros pensadores que abordaron el beneficio de la educación física para la formación humanista. En cuanto a los antecedentes directamente vinculados a la formación de los profesionales de la Cultura Física, existe una tendencia a reiterar tres hechos históricos del siglo XIX: el viaje a Madrid de O'Gavan para estudiar la pedagogía pestalozziana, la fundación del primer gimnasio y la obra de Agüera. Se ha insistido tanto que tal parece que no existieran otros, pero algunos libros de texto relacionados con la enseñanza, artículos en publicaciones seriadas de la época y otros hechos de relevancia histórica demuestran lo contrario. Lo anterior conspira contra el estudio de las formas adoptadas por la formación humanista en el desarrollo del pensamiento cubano y sus nexos con la Cultura Física.

Los sistemas pedagógicos desarrollados en Europa en la primera década del siglo XIX acentuaban cuán necesaria era la educación Física para alcanzar la integralidad de la formación humanista. Muchos pensadores del siglo XIX cubano recibieron una gran influencia de estos modelos pedagógicos y apoyaron la idea de incorporar la Educación Física a la formación en los centros educativos, como vía para mejorar así el rendimiento intelectual. Como asegura Alfonso (2009), entre las personalidades sobresalientes en este sentido cabe citar a Ruiz, Castro y Agüera. En este período se fundaron gimnasios y los pedagogos cubanos reflexionaron sobre la importancia y la necesidad de la actividad física para la formación, a partir de la articulación entre la formación intelectual, moral y física. Al analizar la obra pedagógica de estos pensadores se puede profundizar en el proceso configurativo de la Cultura Física como dimensión importante para la formación humanista.

Entre 1807 y 1808, O'Gavan permaneció en España bajo el patrocinio de la Sociedad Económica de Amigos del País. El viaje tenía la finalidad de estudiar el método pestalozziano, así como las implicaciones del mismo en el contexto educacional y cultural cubano. Allí constató las notables diferencias entre este método y el escolástico, habitualmente empleado en la enseñanza. Hasta ese momento, la educación se limitaba a lo puramente intelectual y espiritual.

Después del periplo por España, O'Gavan redactó un informe que presentó a la Real Sociedad Patriótica de Amigos del País, en el que proponía incorporar la gimnasia a los planes de estudio de la Educacion Fisica. No obstante, la propuesta no fue aceptada por las autoridades pertinentes. Fue el iniciador de la Educación Física, asentada sobre los criterios y las tendencias modernas del pensamiento filosófico de la época, fruto de sus estudios del método de Pestalozzi y de su evaluación crítica de las ventajas y las limitaciones del mismo. Esto ha hecho que algunos autores lo consideren el padre de la Educación Física en Cuba, aunque no se dedicó plenamente a ella.

El Plan de Instrucción Pública, presentado por Ruiz, el 18 de octubre de 1817, lo situó entre los exponentes de las ideas pedagógicas más avanzadas de la época (Alfonso, 2009). Inspirado en las ideas renacentistas del humanista Feltre, las revolucionarias de Rousseau, de Buchan y otros, reprochó a la educación de su tiempo el no promover "el ejercicio corporal". Habría que esperar hasta 1892 por la introducción de la Gimnasia como asignatura en los planes de estudio en las escuelas normales, y a 1900 por la creación de un gimnasio en los institutos de segunda enseñanza, gracias a la Orden Militar 267. Ruiz también planteó la posibilidad de incluir el ejercicio físico en la educación. Sus ideas, aunque aceptadas por 
algunos, no fueron aprobadas por otros. El entonces secretario de la comisión evaluadora de la propuesta realizada por Ruiz fue O'Gavan fue quien impidió se llevara a cabo tan revolucionaria propuesta.

España ejerció una considerable influencia en el posterior desarrollo de las actividades físicas en Cuba. El deseo de renovar el programa educativo e incrementar el desarrollo de las facultades intelectuales y creativas a partir de las ideas de Amorós hizo que la clase acomodada y los españoles residentes en el país respaldaran la proposición de Castro en la Sección de Educación de la Sociedad Económica de Amigos del País, en 1839. Allí presentó un informe titulado "Escuela Gimnástica", que proponía incluir la Gimnasia en la formación de la juventud. Luz y Caballero y Del Monte secundaron con particular fuerza este criterio.

Gracias a estos sucesos comenzó la era de los gimnasios, con la fundación del primero en La Habana. Su creación, no obstante, es controversial. Unos lo adjudican a O'Gavan, por el informe defendido ante los miembros de la Sociedad Económica de Amigos del País. Al parecer, por su oposición al plan educacional de Ruiz, es muy posible que la defensa del informe influyera en la autorización para la construcción del primer gimnasio. Los lineamientos generales del mismo obedecieron a los principios del español Amorós, considerado entre los fundadores de la Educación Física moderna (Martínez de Osaba, 2006).

Otros atribuyen la fundación del mismo a un documento escrito por Castro, registrado en las memorias de la Sociedad Económica de Amigos del País de La Habana, que proponía la construcción de un gimnasio. Lo cierto es que en 1839 se comenzó a construir y en 1843 se inauguró como el Primer Gimnasio Normal, dirigido según los principios pedagógicos de Amorós. Este primer gimnasio inspiró la creación de otros, sobre todo porque quienes estudiaron en Francia fueron influenciados por el método de este. El estudio de los principios y las regularidades de la actividad física en Cuba se inició con la creación de estos gimnasios.

Otro precursor fue Agüera, autor de Gimnástica Moderna o de Consulta, quien brindó una atención adecuada a la práctica de esta disciplina deportiva, y fue uno de sus mayores exponentes en el colegio de los padres escolapios en Guanabacoa. Entre los aportes de Agüera cabe mencionar la fundación de la Escuela de Educación Física para los Bomberos de Cuba y la creación de un sistema de preparación física.

\section{Contribución de la emigración cubana a la inclusión del deporte en el país}

En el siglo XIX, la emigración de cubanos a los EE.UU. y Europa influyó también en el desarrollo de la Cultura Física. En Estados Unidos conocerían varios deportes: béisbol, voleibol y baloncesto; y en Europa, esgrima y gimnasia. A finales del siglo XIX, la Educación Física se desarrolló considerablemente en Cuba, lo cual se evidencia en la inclusión de la gimnasia como asignatura en los programas de la enseñanza media en 1897.

La esgrima como deporte organizado surgió a fines de las década del sesenta del siglo XIX, con la inauguración de la sala de armas del Casino Español. El italiano Galletti fue el primer director, hasta 1874, cuando lo sustituyó el francés Cherembau. La finalidad de la práctica de este deporte era, en un primer momento, velar por la "justicia", pero con el tiempo ganó adeptos, por ser un medio esencial para el desarrollo de músculos y destrezas.

El 20 de diciembre de 1878 se fundó la primera institución estrictamente organizativa, la entidad deportiva nombrada Liga Profesional de Beisbol en La Habana. Esta entidad estaba bajo el auspicio del señor Leopoldo de Sola, y su objetivo fundamental era la práctica del beisbol, con la participacion de tres equipos: Habana Baseball Club, Almendares Baseball 
Club y Matanzas Baseball Club. En 1884 se crea el Club Náutico de Santiago de Cuba y en 1885 el Havana Yacht Club. Ambas instituciones fueron asociaciones con una gran influencia en el desarrolllo deportivo (Alfonso, 2009).

\section{DISCUSIÓN Y POSIBLES LÍNEAS DE INVESTIGACIÓN}

En la actualidad, es insuficiente la investigación referida a la Educación Física y el deporte en Cuba durante el siglo XIX en archivos, bibliotecas y otras organizaciones culturales. Si a esto se añade la diversidad de fuentes, en ocasiones, en mal estado de conservación, se comprende el verdadero desafío de acceder a ellas y resumir la esencia del origen y la difusión de la Cultura Física en Cuba durante ese siglo y principios del siglo XX para compilar conocimientos sobre hechos socioculturales.

Otro de los problemas teóricos para el estudio de la Historia de la Cultura Física y, por tanto, de la formación de los profesionales, es la utilización de diferentes términos, lo que provoca una gran discordancia teórica. Los autores usan indistintamente "gimnasia", "calistenia", "ejercicios corporales", "ejercicio físico", "deporte”, "educación física” y "juego", en todo el siglo XIX y principios del siglo XX. Las futuras investigaciones deben encaminarse a la recuperación de la memoria histórica de la Cultura Física en Cuba, a partir de esta revisión, podrían abordar los siguientes temas:

- La contribución de las ciencias en el desarrollo de la Educación Física.

- El mejoramiento y la creación de nuevos espacios escolares y sociales destinados a la educación física y al deporte (gimnasios, clubes, ligas y federaciones).

- La tradición militar y la institucionalización de la educación físico-deportiva.

- Las primeras competiciones atléticas escolares como elemento dinamizador.

En el mundo actual se debe evitar la pérdida de la memoria histórica. Por tanto, es sumamente importante, en la formación de los profesionales del área, adquirir conocimientos históricos sobre su futura profesión, no solo a nivel mundial, sino también nacional y local. Estos profesionales deben adquirir un conocimiento profundo de la Cultura Física desde sus diferentes perspectivas $y$, en este sentido, las Ciencias Sociales contribuyen significativamente a la formación integral de estos profesionales.

Las Ciencias Sociales aplicadas a la Cultura Física preparan para una vida dinámica, incrementando las potencialidades humanas, para que la vida profesional esté signada por una profunda formación humanista que le permita al futuro licenciado en Cultura Física conducirse con éxito; por tanto, conocer sobre la historia de su profesión enriquece sus conocimientos acerca de los disímiles principios filosóficos que sustentan todo el devenir histórico de la Cultura Física. La adquisición de los conocimientos que se aluden anteriormente lo ayudarán a participar activamente en la construcción una nueva sociedad, que exigen los tiempos actuales; en especial si en su práctica profesional demuestra una formación integral.

\section{CONCLUSIONES}

El pensamiento cubano del siglo XIX tiene un significado especial para la formación, por su atinada contribución al humanismo. En los materiales revisados se valora la pedagogía como ciencia con un hondo sentido humanista, pues la formación del hombre y la 
transformación social están entre sus fines, y se observa una estrecha relación con la Cultura Física. La pedagogía se concibe como ciencia que ubica al ser humano en el centro del proceso formativo, sin dejarse atrapar en el laberinto descontextualizado de los cánones o modelos foráneos extrapolados a la realidad cubana. Incluso se confirma, en algunos pensadores, no solo esa estrecha relación con la Cultura Física, sino también una apología de la misma, traducida en hechos que contribuyeron a su génesis y desarrollo. Sin embargo, se constata que aún son insuficientes los estudios históricos que evidencien documentalmente diferentes hechos estrechamente relacionados con la Educación Física y deporte. Por tanto, es importante la recuperación de la memoria histórica de la Cultura Física en Cuba para formar una conciencia histórica adecuada en los profesionales. Este preámbulo sienta las bases para el desarrollo de futuras investigaciones que contribuyan a la formación de los futuros licenciados en Cultura Física.

\section{REFERENCIAS}

Alfonso, J. (2009). Apuntes para la Historia de la Cultura Física. La Habana: Deportes.

De la Luz y Caballero, J. (2001a). Obras (Vol. I). La Habana: Imagen Contemporánea.

De la Luz y Caballero, J. (2001b). Obras (Vol. II). La Habana: Imagen Contemporánea.

De la Luz y Caballero, J. (2001c). Obras (Vol. III). La Habana: Imagen Contemporánea.

Guadarrama, P.M. (2001). Humanismo y autenticidad en el pensamiento filosófico latinoamericano. Islas 43(128), 87-122. Recuperado de: http://islas.uclv.edu. cu/index.php/islas/article/view/744/654

Mariño, O. (2011). Visión histórica del deporte en Cuba (1762 - 1961). La Habana: Deportes.

Martínez, J. A. (2006). Cultura Física y Deportes. Génesis, evolución y desarrollo (Hasta la Inglaterra del siglo XIX). La Habana: Deportes. Recuperado de https://www.ecured.cu/Cultura f\%C3\%ADsica y deportes G\%C3\%A9nesis, evoluci \%C3\%B3n y desarrollo (Hasta la Inglaterra del siglo XIX) (Libro)

Masjuan, M. A. (1984). El deporte y su historia. La Habana: Científico-técnico.

Rodríguez-González, R. (2010). La significación social de la Cultura Física en la pedagogía humanista de José Martí. Odiseo, 8(15). Recuperado de: http://www.odiseo.com. $\underline{\mathrm{mx} / 2010 / 8-15 / \text { rodriguez-cultura-fisica-marti.html }}$

Rodríguez-González, R. (2011a). Formación humanista y cultura física en la pedagogía del italiano Vittorino Rambaldoni da Feltre. Odiseo, 8(16). Recuperado de: http://www.odiseo.com.mx/bitacora-educativa/2011/02/formacion-humanista-culturafisica-pedagogia-italiano-vittorino-rambaldon

Rodríguez-González, R. (2011b). Formación humanista y cultura física en la pedagogía del médico italiano Hyeronimus Mercurialis. Odiseo, 8(16). Recuperado de: http://www.odiseo.com.mx/bitacora-educativa/2011/05/formacion-humanista-culturafisica-pedagogia-medico-italiano-hyeronimus-m

Rodríguez-González, R. (2016). Estrategia de formación humanista-martiana en la licenciatura en Cultura Física. Apunts. Educación física y deportes, 125. Recuperado de: http://www.revista-apunts.com/es/hemeroteca?article $=1752$ 
Saco, J. A. (2001). Obras. La Habana: Imagen Contemporánea.

Salas, J. A. (2009). Génesis y difusión de la educación física en Cuba: (1800-1901) (Tesis de Doctorado, Universidad de Salamanca). Recuperado de: http://hdl.handle.net/10366/76296

Sánchez, B. P. (2004). Cultura Física y Epistemología: una propuesta para el estudio de la experiencia cubana (Tesis en opción al Grado Científico de Doctor en Ciencias de la Cultura Física). Instituto Superior de Cultura Física "Manuel Fajardo", Cuba.

Sosa, E., \& Penabad, A. (2005a). Historia de la educación en Cuba. La Habana: Pueblo y Educación.

Sosa, E., \& Penabad, A. (2005b). Historia de la educación en Cuba. La Habana: Pueblo y Educación.

Torres, E. (2006a). En busca de la cubanidad (Vol. I). La Habana: Ciencias Sociales.

Torres, E. (2006b). En busca de la cubanidad (Vol. II). La Habana: Ciencias Sociales.

Vives, J.L. (1988). Diálogos y otros escritos. Recuperado de https://www.casadellibro. com/libro-dialogos-y-otros-escritos/9788432039805/19667

Vives, J. L. (1994). De institutione feminae christianae. Recuperado de https://books. google.co.cr/books/about/De Institutione Feminae Christianae.html?id=VfQpAAAAYA AJ\&redir esc $=y$

Vives, J. L. (1997). Las Disciplinas. Recuperado de https://www.casadellibro.com/libro-lasdisciplinas/mkt0003325980/4599511

Varela y Morales, F. (2001a). Obras (Vol. I). La Habana: Imagen Contemporánea.

Varela y Morales, F. (2001b). Obras (Vol. II). La Habana: Imagen Contemporánea.

Varela y Morales, F. (2001c). Obras (Vol. III). La Habana: Imagen Contemporánea. 